\title{
Pattern and characteristics of patients admitted to a hospice connected with an acute palliative care unit in a comprehensive cancer center
}

\author{
Sebastiano Mercadante ${ }^{1}$ (D ) Fausto Giuliana ${ }^{1} \cdot$ Rosanna Bellingardo ${ }^{1} \cdot$ Gianluca Albegiani $^{1} \cdot$ Grazia Di Silvestre $^{2}$. \\ Alessandra Casuccio ${ }^{3}$
}

Received: 29 April 2021 / Accepted: 7 November 2021 / Published online: 29 November 2021

(c) The Author(s), under exclusive licence to Springer-Verlag GmbH Germany, part of Springer Nature 2021

\begin{abstract}
Purpose Information about inpatient hospice activity is limited. No data exist about the pattern and the characteristics of advanced cancer patients admitted to a hospice connected to an acute supportive/palliative care unit (ASPCU).

Methods Data of hospice admissions were retrieved from the database where all data were prospectively collected. The Edmonton Symptom Assessment Scale (ESAS) and the use of analgesics and adjuvant were recorded at admission (T0), 1 week (T7), 2 weeks (T14), and the day before death (T-end). The use of palliative sedation and its indication, duration, and drugs end doses used were recorded. The number of hospice deaths, discharges, and hospice staying were recorded.

Results One hundred seventy-seven patients were admitted in 13 months. There were significant differences in total ESAS at T0 $(P=0.033)$, total ESAS being significantly lower in patients admitted from the ASPCU than those referred from other settings. The day before death (T-end), only 48 patients could be evaluated. Patients referred by a palliative care setting were more likely to be prescribed opioids at T0 $(P=0.0007)$. At T-end, there was a significant increase in the use of morphine and haloperidol $(P<0.05)$. Seventeen percent of patients died within $48 \mathrm{~h}$. Only a minority of patients could be properly assessed at T-end (25\%). Palliative sedation was performed in $10.1 \%$ of patients. The mean hospice staying was 16.3 (SD 21.4) days. There were no differences in mean hospice staying between patients who died in hospice or those discharged $(P=0.873)$.

Conclusion The presence of a hospice in a comprehensive cancer center could offer a further opportunity for continuing care. Specialized palliative care may be offered to patients referred from other hospitals, home palliative care, but above all, transfer to hospice may allow a continuity of care for those patients who were initially admitted to an ASPCU for symptom control, to which anticancer therapies were withdrawn or withhold after multidisciplinary consultation. Similarly, after a proper palliative care consultation in other hospital units, patients may be referred to hospice. This process may avoid transfers to external hospices, which can prevent the continuity of care.
\end{abstract}

Keywords Palliative care $\cdot$ Hospice $\cdot$ Advanced cancer

Sebastiano Mercadante

terapiadeldolore@lamaddalenanet.it; 03sebelle@gmail.com

1 Mean Regional Center for Pain Relief and Palliative Care Unit, La Maddalena Cancer Center, Via San Lorenzo 312, 90146 Palermo, Italy

2 Palliative Care Center, ASP Palermo, Palermo, Italy

3 Department of Health Promotion, Mother and Child Care, Internal Medicine and Medical Specialties (PROMISE), University of Palermo, Palermo, Italy

\section{Introduction}

At the end of seventies, palliative care was initiated in Italy as a natural progression from an initial interest in cancer pain management to a growing need to resolve the complex problems of advanced cancer patients. About 10 years after, various organizations developed palliative care programs, principally based on home palliative care for patients with very advanced stage disease [1]. In the last decades, the Health Care System has allocated specific resources in the field of community care and health districts under the input of new legislations. As a consequence, several centers involved in palliative care progressively increased, 
particularly concentrated in Northern Italy, and more than 300 hospices are now available for palliative care population [2]. In pioneer studies, despite being referred to hospices, more than $30 \%$ of patients with advanced cancer reported that they suffered from unacceptably severe pain [3].

Patients with advanced cancer admitted to hospice continue to experience distress from numerous symptoms [4]. Although hospice patients experienced significantly less depression and expressed more satisfaction with care in comparison with conventional care, hospice care has been reported to offer only minimal differences in terms of cost or effectiveness. No differences in outcomes were found between the two groups including costs, length of hospital stay, pain, survival, use of major surgical procedures, chemotherapy, and activities [5]. A meta-analysis demonstrated a small benefit on patients' pain, other symptoms, and a non-significant trend towards benefits for satisfaction and therapeutic interventions [6].

In the last decade, there has been a rapid growth in the number and quality of hospices, possibly providing better outcomes with more professional knowledge, experience, and advanced clinical approach. In Italy, the hospice is accessed by patients suffering an evolving progressive disease no longer susceptible to active therapy and is evaluated according to some criteria that include life expectancy, usually assessed in 6 months, or having an incurable disease that will inevitably lead to premature death, even if in an extremely variable and not always easily predictable time. Other criteria include high level of need for assistance and/or reduced functional autonomy and/or impairment of the cognitive state characterizing a state of "fragility" and inability to implement palliative home care, due to health or social problems. Patients can access the services guaranteed by hospitalization in hospice from any care setting, as long as they are consistent with the specific requirements indicated above. The hospice is accessed through a multidimensional assessment process that verifies the possession of the required requirements and priority and allows the identification of the most appropriate methods of care for each patient and his family [2].

Data regarding the clinical activity in traditional hospices has been variably reported, but information is scarce. In November 2019, a new hospice was launched at a comprehensive cancer center. Differently from other hospices, however, this inpatient unit has joined a pre-existing 10 beds of acute supportive/palliative care unit (ASPCU) in the same hospital to provide continuity of care for patients who were no longer considered for anticancer therapies [7]. Differently from typical hospices, patients are admitted to this acute unit for distressing symptoms, particularly pain, severe toxicity from treatments, or derangement of the general conditions, re-assessment of prognosis to delineate the proper pathway of care, that is, continuing anticancer treatment or transition to palliative care, home care, or hospice, according to individual situations. In APSPCU the mortality rate is low (about 5\%). In other words it is a bridge between oncology and palliative care. This has a relevant influence on the prevention of aggressive chemotherapy in the last months of life $[8,9]$. The ASPCU activity is reimbursed by DRG (disease-related group) by the Regional Health Care Service, while hospice activity is reimbursed on daily allowance [10]. The aim of this study was to assess the pattern and the characteristics of advanced cancer patients admitted to hospice, recently added to enrich the offer of palliative care in a comprehensive cancer center.

\section{Methods}

\section{Structure}

The hospice is traditionally equipped with single bed rooms, with facilities for allowing caregivers to stay, and dedicated trained personnel. It is based on the same floor next to preexisting ASPCU, equipped with 10 beds for complex cases and early palliative care for patients who mainly receive or recently received anticancer treatments. The activity of this unit has been described elsewhere [7]. The main differences between the two units are listed in Table 1. Physicians regularly rotate their activity between the two units, ASPCU and hospice, with frequent meetings to exchange information and to share decision-making process. Consultations are available for most specialties, as well as other facilities of the hospital (biochemistry, imaging studies, and so on).

\section{Population}

A consecutive sample of patients admitted in a period of 13 months (from November 11, 2019, to December 10, 2020) was taken into consideration. In this period, we afforded the COVID-19 crisis, which imposed some restrictions in admission and family presence, although, first in Italy, we adopted a protocol to facilitate the presence of the caregiver [9].

\section{Measurements}

Data of hospice admissions were retrieved from the database where all data were prospectively collected every day. The following parameters were drawn: age, gender, diagnosis, and performance status; referral (primary care, home palliative care, acute hospital, and others units), reasons for admission, anticancer therapies in the previous 15 days, and the use of analgesics and co-analgesics. The Memorial Delirium Assessment Scale (MDAS), a screening test for history of alcohol dependence (CAGE: cut down, annoy, 
Table 1 Admission criteria and main objective for admission in ASPCU and hospice

\begin{tabular}{lll}
\hline Characteristics and admission criteria & Objectives \\
\hline ASPCU & - Patients with cancer & - Rapid pain and symptom stabilization \\
& - Patients receiving anticancer therapy presenting pain and symptoms, or worsening of & - Selection of patients for transition of care \\
& clinical condition & \\
- Patients with symptoms who need a reassessment for a possible transition of care (pal- & \\
& liative care only) & \\
- Patients diagnosed with cancer but strongly symptomatic for starting chemotherapy & \\
- Admission on emergency from other wards or other hospitals & - Control of pain and symptoms after sta- \\
- DRG reimbursement & bilization (if transferred from ASPCU) \\
- Short staying & - Helping patients and families with social \\
- Low mortality rate (about5\%) (7,10,16) & and psychological support \\
Hospice & - Patients with advanced cancer or non-cancer progressive diseases with limited survival \\
& not receiving active therapies & \\
- Patients who cannot be followed at home for social or clinical problems & \\
- Respite admission for relieving caregiver burden & \\
- Long staying (up to 6 months) & \\
- Daily reimbursement & High mortality rate
\end{tabular}

guilt, eye-opener) [11], or drugs were assessed (CAGE for drugs). MDAS is a 10-item clinician-rated assessment scale that has been validated for the assessment of delirium in cancer patients. It measures the level of consciousness, disorientation, memory, recall, attention, disorganized thinking, perceptual disturbance, delusions, psychomotor activity, and sleep The items are scored between 0 and 3, for a total score from 0 to 30 [12]. According to our experience, a value of $>7$ suggest an initial cognitive disturbances, while values $>13$ mean severe cognitive failure [13]. CAGE is considered to be positive by "yes" answer to at least two of questions for men and at least one for women [13]. When patients were unable to complete the assessment tools, nurse and caregivers filled in the data in the chart, when possible.

The Edmonton Symptom Assessment scale (ESAS) and the use of analgesics and adjuvant were recorded at admission (T0), 1 week (T7), 2 weeks (T14), and the day before death (T-end). The ESAS is a widely used validated tool to measure the severity of common physical and psychological symptoms (pain, weakness, nausea, depression, anxiety, drowsiness, dyspnea, poor appetite, poor sleep, and poor feeling of well-being) on a numeric rating scale ranging from 0 to 10 [14].

The use of palliative sedation and its indication, duration, and drugs end doses used were recorded. Palliative sedation was used to reduce the level of consciousness in patients with refractory symptoms in the last days of life. The number of hospice deaths, discharges, and hospice staying were recorded.
The Institutional Review Board of ASP Palermo 6 approved the study, and patients or surrogates gave their informed consent.

\section{Statistical analysis}

Continuous variables are presented as mean (SD), and categorical variables are expressed as number of patients (percentage). Chi-square or Fisher's exact tests were used for categorical variables, as appropriate, and the univariate analysis of variance (ANOVA) test was performed to evaluate the mean differences between patient groups. The repeated measures ANOVA test was used to compare continuous variables at different time intervals. The paired Wilcoxon signed-rank test was used to compare symptom intensity scores and drug use differences in the four time intervals. The data were analyzed by the SPSS software, version 22 (SPSS Inc., Chicago, IL, USA). All statistical tests were two-tailed, and statistical significance was defined as $P \leq 00.05$.

\section{Results}

One hundred seventy-seven patients were admitted in the period taken into consideration. Eighty-eight patients (49.7\%) were males, and the mean age was 68.6 years (SD 11.6). The mean Karnofsky at admission was 31 (SD 10). The mean Karnofsky was significantly higher in patients who were referred from a palliative care setting (33.2, SD 9.5 versus 26.9, SD 9.6, $P=0.0005$ ). Five patients 
Table 2 Characteristics of patients

\begin{tabular}{|c|c|}
\hline Uncontrolled pain & $88(49.7 \%)$ \\
\hline Symptom control & $118(66.6 \%)$ \\
\hline Respiratory & $60(33.9 \%)$ \\
\hline Gastrointestinal & $24(13.6 \%)$ \\
\hline Neurological & $31(17.5 \%)$ \\
\hline Psychiatric & $3(1.7 \%)$ \\
\hline Other & $3(1.7 \%)$ \\
\hline \multicolumn{2}{|l|}{ Social } \\
\hline $\mathrm{N}^{\circ}$ patients & 177 \\
\hline Gender (male/female) & $88(49.7 \%) / 89(50.3 \%)$ \\
\hline Age, mean(SD) yrs & $68.6(11.6)$ \\
\hline Karnofsky, mean(SD) & $31(10)$ \\
\hline \multicolumn{2}{|l|}{-Primary diagnosis } \\
\hline - Cancer & 153 \\
\hline Gastrointestinal & 53 \\
\hline Lung & 34 \\
\hline Genitourinary & 23 \\
\hline Breast & 15 \\
\hline Hematologic & 14 \\
\hline Head-neck & 9 \\
\hline Others & 5 \\
\hline -Non cancer diseases & 24 \\
\hline Neurologic disease & 13 \\
\hline Lung disease & 6 \\
\hline Cardiac disease & 4 \\
\hline Infection & 1 \\
\hline \multicolumn{2}{|l|}{ Referral } \\
\hline \multicolumn{2}{|l|}{ Territory: } \\
\hline Home palliative care & 23 \\
\hline \multicolumn{2}{|l|}{ Hospital Units: } \\
\hline ASPCU & 93 \\
\hline Oncology & 11 \\
\hline Hematology & 3 \\
\hline Surgery & 3 \\
\hline Other & 7 \\
\hline Other hospitals: & 35 \\
\hline \multicolumn{2}{|l|}{ Education } \\
\hline Primary & $32(18.1 \%)$ \\
\hline Lower secondary school & $41(23.2 \%)$ \\
\hline Higher secondary school & $53(29.9 \%)$ \\
\hline Degree & $28(15.8 \%)$ \\
\hline
\end{tabular}

were CAGE-positive for alcohol. One patient was CAGEpositive for drugs. Two patients received anticancer therapy in the last 15 days before hospice admission. Four patients underwent palliative surgery in the last 15 days.
Table 3 Reasons for admission (multiple choice)

\begin{tabular}{ll}
\hline Uncontrolled pain & $88(49.7 \%)$ \\
\hline Symptom control & $118(66.6 \%)$ \\
Respiratory & $60(33.9 \%)$ \\
Gastrointestinal & $24(13.6 \%)$ \\
Neurological & $31(17.5 \%)$ \\
Psychiatric & $3(1.7 \%)$ \\
Other & $3(1.7 \%)$ \\
Social problems & $55(31.1 \%)$ \\
\hline
\end{tabular}

The general characteristics of patients are described in Table 2. Twenty-four admissions (13.5\%) were non cancer patients. Reasons for hospice admission were often mixed and included in a rank order: symptom control, and pain control, as well as social problems (Table 3 ).

\section{Symptoms}

Changes of ESAS items intensity are represented in Table 4. There were significant differences in total ESAS at T0 $(P=0.033)$, total ESAS being significantly lower in patients referred from the ASPCU (29.5, SD 16.6) than in those referred from other wards, hospitals, or home palliative care. Specifically, patients coming from oncology ward had the highest total ESAS at T0 (47.1, SD 12.8). Differences between cancer and non-cancer patients were not evaluated, considering the lower number of patients in the latter group.

There were significant changes in symptom intensity 1 week and 2 weeks after hospice admission, particularly for pain, insomnia, weakness, and well-being, as well total ESAS. The mean MDAS was 14.4 (SD 12.3). Not all patients could report their ESAS, because of their cognitive deterioration (n.115, 64\%). The day before death (T-end), only 48 eight patients could be evaluated.

\section{Drugs}

The use of opioids and adjuvant drugs is reported in Table 5 . Patients referred by a palliative care setting (ASPCU and home palliative care) were more likely to be prescribed opioids at T0 $(P=0.0007)$. At T-end, there was a significant increase in the use of morphine and haloperidol $(P<0.05)$.

\section{End of life issues}

Of 141 patients who died in hospice, 24 of them (17\%) died within $48 \mathrm{~h}$. At admission these patients had lower Karnofsky levels in comparison with the other patients (22.3, SD 9.7 versus $30.9, \mathrm{SD}=9.5, P=0.0005$ ). Only a minority of patients could be properly assessed the at T-end $(n=48$, 
Table 4 ESAS at the different time intervals (mean (SD). Not all patients had a complete evaluation

\begin{tabular}{llllllc}
\hline & T0 & T7 & T14 & T-end & p(T0vsT7) & p(T0vsT14) \\
\hline ESAS & $\mathbf{n . 1 1 5}$ & $\mathrm{n} .79$ & $\mathrm{n} .79$ & $\mathrm{n} .48$ & & \\
Pain & $2.7(2.7)$ & $1.5(2.2)$ & $1.4(2.1)$ & $1.9(2.7)$ & 0.011 & 0.029 \\
Dyspnea & $1.8(2.6)$ & $1.0(2.4)$ & $1.3(2.3)$ & $1.5(2.4)$ & 0.458 & 0.520 \\
Anxiety & $3.0(3.2)$ & $2.1(3.0)$ & $2.8(3.0)$ & $2.2(2.9)$ & 0.217 & 0.434 \\
Depression & $2.9(3.3)$ & $1.7(2.9)$ & $2.2(3.1)$ & $2.1(3.0)$ & 0.347 & 0.352 \\
Insomnia & $3.5(3.6)$ & $1.9(2.8)$ & $2.5(3.4)$ & $2.2(2.9)$ & 0.007 & 0.078 \\
Drowsiness & $3.7(3.0)$ & $2.8(2.7)$ & $3.3(3.2)$ & $3.5(3.3)$ & 0.728 & 0.727 \\
Nausea & $1.8(3.2)$ & $1.0(1.9)$ & $0.8(2.0)$ & $0.5(1.5)$ & 0.118 & 0.032 \\
Poor appetite & $3.8(3.3)$ & $3.1(3.7)$ & $2.5(3.3)$ & $2.4(3.5)$ & 0.860 & 0.784 \\
Weakness & $5.5(3.1)$ & $3.2(3.3)$ & $4.2(3.3)$ & $4.6(3.5)$ & 0.002 & 0.344 \\
Poor well-being & $5.1(2.9)$ & $2.8(3.1)$ & $3.2(3.1)$ & $3.3(3.4)$ & $<0.0005$ & $<0.0005$ \\
Total32.9(18.5) & $19.9(15.9)$ & $22.8(17.7)$ & $23.8(18.8)$ & 0.002 & 0.038 & \\
\hline
\end{tabular}

$25 \%$ ), and no differences in total ESAS among the types of referral were found $(P=0.683)$.

Palliative sedation was performed in 18 patients $(10.1 \%)$. The principal reasons were delirium (n.10), dyspnea (n.5), and existential distress (n.8) (in some cases patients had more reasons for starting palliative sedation). The mean starting doses and the final dose (at T-end) of midazolam were $57.8 \mathrm{mg} /$ day (SD 35.1) and $89.2 \mathrm{mg} /$ day (SD 46.8), respectively.

\section{Hospice staying}

The mean hospice staying was 16.3 (SD 21.4) days (median 11 , interquartile range 6-22). One hundred forty-one patients died in hospice (79.4\%), and 33 patients (18.6\%) were discharged home. There were no differences in mean hospice staying between patients who died in hospice (16.4 days, SD 22.2) and those who were discharged home (15.8 days, SD 17.6) $(P=0.873)$. Patients admitted from ASPCU or home care (palliative care setting) had a longer staying (17.7 (SD 23.1) and 13.6 (SD17.3) days, respectively), although this data did not attain significance $(P=0.232)$.

\section{Discussion}

\section{General aspects}

This study provided interesting information regarding some clinical aspects of patients admitted to a hospice in a comprehensive cancer center with a pre-existing ASPCU, where experience and clinical footprint were shared between the two teams.

First, most patients (2/3) had previously received specialized palliative care, either at home or in the ASPCU (23 and 93 , respectively), with only $1 / 3$ (59 patients) not having consultation or assistance of a palliative care team. Of interest, patients admitted from a palliative care setting had a Karnofsky higher and survived something more than patients admitted from other places. This in contrast with other studies in which most patients were referred from home, possibly without a previous palliative care consultation [15]. Reasons for hospice admission were variable and included about $1 / 3$ of patients who had logistic problems at home, other than clinical problems. Patients who cannot be adequately assisted at home for lack of caregiver or other social problems have a clear indication for hospice admission. In a previous multicenter study performed in Italy, however, social problems were rarely the indication for hospice admission [15]. In Germany, problems and symptoms impairing autonomy and mobility, but not primarily pain or dyspnea, were the main reasons for admission to hospice. The most frequent symptoms/problems were weakness (95\%), need of assistance with activities of daily living (88\%), overburdening of family caregivers (83\%), and tiredness (75\%). Four symptom clusters were identified and included deteriorated physical condition/decompensation of home care, emotional problems, gastrointestinal symptoms, and other symptoms [16]. In the Netherland, with a different cultural profile and hospice typology, hospice application was quite variable. In a large study, most patients were admitted to hospices with a declared wish to die. Hospice typology includes stand-alone hospices, hospice-unit nursing homes, and volunteer-driven hospices, also explaining different levels of expertise [17, 18]. In South Korea, it has been reported that family doctors (dedicated hospice physician group) performed better than oncologists (non-dedicated physician group) [19]. This underlines the huge differences in hospice facilities existing around the world. In Italy hospices are run by a physician with a multiprofessional team, including doctors, nurses, psychologists, social workers, physiotherapists, and other health professionals. The reimbursement provided by the national Health Care System is a fixed amount/day of 250 
Table 5 Drugs administered at the different time intervals. $T D$ transdermal, $O$ oral, $S C$ subcutaneous, $I V$ intravenous. Percentage in bracket

\begin{tabular}{|c|c|c|c|c|}
\hline \multicolumn{5}{|l|}{ Drugs } \\
\hline & T0 & $\mathrm{T} 7$ & $\mathrm{~T} 14$ & T-end \\
\hline \multicolumn{5}{|l|}{ Analgesics } \\
\hline TD buprenorphine & $12(6.8)$ & $6(7.8)$ & $2(2.6)$ & $6(12.6)$ \\
\hline TD fentanyl & $20(11.3)$ & $16(20.8)$ & $8(10.4)$ & $14(29.4)$ \\
\hline O-hydromorphone & $2(1.1)$ & $2(2.6)$ & $3(1.7)$ & $1(2.1)$ \\
\hline O-IV methadone & $10(5.6)$ & $6(7.8)$ & $6(7.8)$ & $11(23.1)$ \\
\hline O-IV morphine & $53(29.9)$ & $41(53.3)$ & $21(27.9)$ & $48(100)$ \\
\hline O-oxycodone & $10(5.6)$ & $4(5.2)$ & $3(3.9)$ & $11(23.1)$ \\
\hline O-tapentadol & $2(1.1)$ & - & $1(1.3)$ & $1(2.1)$ \\
\hline O-ibuprofen & $5(2.8)$ & $4(5.2)$ & $4(5.2)$ & $4(8.4)$ \\
\hline O-IV ketorolac & $3(1.6)$ & $4(5.2)$ & $2(2.6)$ & $1(2.1)$ \\
\hline O-IV paracetamol & $14(7.7)$ & $12(15.6)$ & $11(14.3)$ & $9(18.9)$ \\
\hline \multicolumn{5}{|l|}{ Rescue pain medications } \\
\hline IV methadone & $2(1.1)$ & $3(3.9)$ & $3(3.9)$ & $3(6.3)$ \\
\hline Sublingual fentanyl & $11(6.2)$ & $8(10.4)$ & $5(6.5)$ & $6(12.6)$ \\
\hline IV morphine & $98(55.4)$ & $57(74.1)$ & $28(36.4)$ & $48(100)$ \\
\hline Oral morphine & $13(7.4)$ & $11(14.3)$ & $5(6.5)$ & $9(18.9)$ \\
\hline Oral oxycodone & $2(1.1)$ & $1(1.3)$ & $2(2.6)$ & $6(12.6)$ \\
\hline Oral tramadol & $3(1.8)$ & $1(1.3)$ & - & $1(2.1)$ \\
\hline Nasal fentanyl-pectin & $1(0.6)$ & - & - & - \\
\hline IV Ibuprofen & $1(0.6)$ & $1(1.3)$ & $1(1.3)$ & - \\
\hline IV ketorolac & $1(0.6)$ & - & - & $1(2.1)$ \\
\hline IV Paracetamol & $11(6.2)$ & $8(10.4)$ & $4(5.2)$ & $8(16.8)$ \\
\hline IV Tramadol & $3(1.6)$ & $3(3.9)$ & $2(2.6)$ & $1(2.1)$ \\
\hline \multicolumn{5}{|l|}{ Adjuvants } \\
\hline O-valproate acid & $3(1.8)$ & $2(2.6)$ & $1(1.3)$ & $3(6.3)$ \\
\hline O-SC haloperidol & $22(13.2)$ & $20(26.0)$ & $8(10.4)$ & $44(92.4)$ \\
\hline O-alprazolam & $2(1.1)$ & $1(1.3)$ & $1(1.3)$ & $1(2.1)$ \\
\hline O-bromazepam & $5(3.0)$ & $3(3.9)$ & $2(2.6)$ & $1(2.1)$ \\
\hline $\begin{array}{l}\text { O-IV chlormetaproma- } \\
\text { zine }\end{array}$ & $1(0.6)$ & $1(1.3)$ & $1(1.3)$ & - \\
\hline IV chlorpromazine & $4(2.4)$ & $1(1.3)$ & $5(6.5)$ & - \\
\hline O-clotiazepam & $1(0.6)$ & $1(1.3)$ & $1(1.3)$ & $2(4.2)$ \\
\hline SC-phenobarbital & $3(1.8)$ & $2(2.6)$ & - & $4(8.4)$ \\
\hline O-levetiracetam & $4(2.4)$ & $3(3.9)$ & $3(3.9)$ & $2(4.2)$ \\
\hline O-levomepromazine & $3(1.8)$ & $3(3.9)$ & $2(2.6)$ & $1(2.1)$ \\
\hline O-lorazepam & $2(1.1)$ & $1(1.3)$ & $1(1.3)$ & - \\
\hline IV-midazolam & $10(6.0)$ & $8(10.4)$ & $4(5.2)$ & $25(52.5)$ \\
\hline O-mirtazapine & $1(0.6)$ & $1(1.3)$ & - & $1(2.1)$ \\
\hline O-olanzapine & $12(7.2)$ & $9(11.7)$ & $6(7.8)$ & $9(18.9)$ \\
\hline IV-promazine & $15(9.0)$ & 15 (19.5) & $11(14.3)$ & $18(37.8)$ \\
\hline O-quetiapine & $1(0.6)$ & $2(2.6)$ & $2(2.6)$ & $1(2.1)$ \\
\hline O-risperidone & $1(0.6)$ & $1(1.3)$ & - & - \\
\hline O-triazolam & $1(0.6)$ & - & - & - \\
\hline O-zolpidem & $1(0.6)$ & $1(1.3)$ & $1(1.3)$ & $1(2.1)$ \\
\hline
\end{tabular}

euros [2]. Other structures, such as residential places or nursing homes, are out of the palliative care network.

A low number of patients had a non-cancer disease. Of interest, in a previous multicenter study performed 10 years ago, almost all patients had a diagnosis of cancer [16]. Despite the growing increase of non-cancer incurable diseases, this population is still under-represented in the hospice setting [15]. Similar percentages $(13,6 \%)$ were found in a large multicenter study performed in Germany [16]. On the other hand, this data was expected, considering that most patients were referred by other acute units of the hospital, which is a cancer center.

The short time of hospice staying or time to death, however, imply an inacceptable timing for patients with several problems presumed to be present early during the course of disease, at least for patients referred from other settings. In this study, a large amount of patients had received palliative care either at home or in the ASPCU. In this unit, oncologists refer their patients early in the trajectory of disease when they are receiving active therapy, for pain and symptom control, extreme toxicity, and re-evaluation for gradual transition of care which includes a huge effort of communication and psychological support for both patients and relatives [7]. Indeed, an extremely late referral or multiple hospital admissions in the last 3 months of life indicates a missed opportunity for timely intervention by the palliative care teams designed to address the physical, psychological, social, emotional, and spiritual aspects of care for patients with serious illnesses. This aspect was also underlined in very specialized centers in other countries [20].

\section{Symptoms}

Patients had a consistent symptom burden at admission, although differences between cancer and non-cancer patients were not measurable, because of the unbalanced number of patients in these groups. However, a previous study showed similar symptom burden in patients with malignant and nonmalignant disease [21].

After admission there was a clear improvement of physical and psychological symptoms, as it often occurs in acute or home care settings [22, 23], probably as a result of a comprehensive assessment and team experience in symptom control. In a retrospective study, admission to hospice led to a significant reduction of pain intensity, particularly in patients with moderate to severe pain [24]. It is likely that hospice policies are largely different among countries and even in the same country. It has been reported that a systematic pain assessment and management were performed in only one-third of patients, a percentage that could be much improved in clinical practice [25]. For example, the need of an appropriate clinical documentation is of paramount importance for an appropriate management of palliative care 
patients. In a study in the Netherland, collecting documentation was found to be difficult and time-consuming. Various types of hospice patient records were found with different availabilities, with nurses' records being largely available (98\%), compared to volunteers' records (62\%). Information about the illness was largely available (97\%), but symptoms assessment was poorly available (10\%). The principal barriers were ethical issues, lack of knowledge, and lack of communication [17]. Indeed, specialistic assessment and treatment are the two main steps for the management of patients admitted to hospice.

\section{Drugs}

A large number of patients referred by a palliative care setting were already receiving opioids at hospice admission. Haloperidol and continuous intravenous morphine were the most frequent drugs used in the last days of life. Of interest, most patients had an intravenous line for the treatment of emergencies, particularly those admitted from the ASPCU, most of them already having a peripheral-central catheter. In a previous study, the percentage of patients prescribed given around-the-clock opioids increased from 63 to $76.8 \%$ on the second day and to $89.9 \% 1$ day before death. The most frequent route of drug delivery was the subcutaneous one [26]. In another study performed in Finland the number of patients admitted to hospice opioid doses were increased during their hospice stay [27].

In a similar study, appropriate drugs were found to be more likely to be given continuously in the hospice than in a hospital setting. Opioids were delivered continuously at over twice the rate in hospice than in hospital. These drugs were less frequently started in the last 3 days of life in hospice than in hospital (20.8\% and $13.3 \%$, respectively). Midazolam, haloperidol, and drugs for pulmonary secretions were more frequently started in the final 3 days in hospice than in hospital [14]. Of concern, in the hospital setting, patients were more likely to receive more inappropriate drugs than appropriate drugs in comparison with the hospice setting. Furthermore, patients in hospital were more likely to undergo diagnostic procedures than hospice patients. There was no difference in chance on receiving two or more therapeutic procedures between hospital and hospice patients. In a retrospective study, admission to a hospice led to a significant reduction in reported pain intensity for the patients. This decrease in pain was accompanied by a significant increase in the use of morphine, especially via parenteral routes, but not by a higher mean equivalent daily dose of oral morphine per patient [24]. Thus, hospice care provides an appropriate drug selection for symptom control in comparison with hospital non-specialized care.

\section{End of life issues}

Less than $80 \%$ of patients died in hospice and a minority (10\%) needed to be sedated at the end of life. This observation is in contrast with the findings of other studies. In a previous study performed in Italy, patients admitted to hospice were unlikely to receive specialized palliative care before hospice admission. Of concern, one patient was transferred from an intensive care unit of another hospital and died some hours after hospice admission. In contrast, some patients were transferred from our surgical unit, after a palliative care consultation which prevented the admission to an intensive care unit where patients would have received a disproportionate and distressing treatment before dying. Although the survival was similar, more patients (86\%) died in hospice and a higher number of patients $(25 \%)$ received palliative sedation [15]. One could argue that in a hospice where most patients are referred from a palliative care setting, there would be a lesser need of palliative sedation, possibly because a better assessment and symptom management, although this statement should be proved in studies an appropriate design. Seventeen percent of patients died within 2 days of admission, and one-third of patients could not be properly assessed, particularly 1 day before death, due to deteriorated cognitive function. In a recent study, $2 \%$ of patients received a palliative care referral less than $24 \mathrm{~h}$ before death. These patients had higher scores for depression, drowsiness, and shortness of breath, meaning that at this stage, patients could be assessed [28]. It is likely that the difference resides in an indirect evaluation by proxies or nurses. In this study, symptom assessment consisted of a subjective measurement of symptom intensity, facilitated by a nurse or physician. In Germany, hospice resulted to be a transition place. After a mean admission time of 10 days, about $21 \%$ were discharged palliative home care, with only about $60 \%$ of patients dying in the ward. Of interest, a minimal amount of patients came back to hospital wards for anticancer therapy. It is evident as admission policies, levels of assessment, and organization are quite different among countries and probably in the same country.

This study had several limitations. First, this study was conducted at a single institution. This is a tertiary care comprehensive cancer center, staffed by a well-established palliative care team, connected to the ASPCU. However, this could be a future model allowing a continuing of care [29], including an ASPCU where admitted for symptom control in patients who are still receiving anticancer therapies, or eventually to allow transition from cancer care to other settings, including home care or hospice, where patients unable to be assisted at home or requiring complex treatments difficult to be performed at home can be more usefully admitted. Noncancer patients seem to be an under-represented population. Application should be based on the need for palliation, irrespective of diagnosis. Finally, there was not a comparative group, for example, a territorial hospice. 


\section{Conclusion}

Many people suffering from life-threatening diseases experience great suffering and need palliative care, a means of alleviating symptoms and improving their quality of life. However, problems including fragmentation of services and late referrals prevent many patients from receiving the appropriate palliative care at the right time. Regretfully, little research is performed in hospice care, also because this has different meanings across the countries. In the authors' opinion, different options should be available for providing palliative care, at least in a comprehensive cancer center, including an ASPCU where difficult symptoms are treated during the course of cancer disease, an outpatient clinic for a proper consultation for ambulatory patients, and hospice and home care for patients who have a shorter survival time. All these services should be coordinated to dictate the timely choices for each individual. Consultations in intensive care units or other wards should be prompted to prevent disproportionate treatments in the dying patient $[29,30]$. The presence of a hospice in a comprehensive cancer center offers many advantages. First, specialized palliative care may be offered to patients referred from other hospitals or home palliative care, as it occurs with other hospices. Indeed, a continuity of care is available for those patients who were initially admitted to an ASPCU, when anticancer therapies are withdrawn or withhold after multidisciplinary consultation. Similarly, after a proper palliative care consultation in other hospital units, patients may be referred to hospice. This process may avoid uncomfortable transfers to external hospices, which can deprive the continuity of care. Comparison among different models of hospice are necessary to establish the best care for patients with irreversible conditions, either in terms of costs or quality.

Author contribution SM, project and writing; FG, data retrievement; $\mathrm{RB}$, data retrievement; GLA, data retrievement; GD, administrative; $\mathrm{AC}$, statistics. The authors approved the text and have full control of all primary data; we agree to allow the journal to review their data if requested.

Data Availability Data available on request.

Code availability Word.

\section{Declarations}

Ethics approval It is a non-interventional study based on routine data recording. EC was deemed to be unnecessary.

Consent to participate See above.

Consent for publication See above.
Conflict of interest The authors declare no competing interests.

\section{References}

1. Mercadante S, Vitrano V (2010) Palliative care in Italy: problems area. Minerva Anestesiol 76:1060-1071

2. Zucco F. (2007) Hospice in Italia, Ministero della Salute,

3. McMillan S (1996) Pain and pain relief experienced by hospice patients with cancer. Cancer Nurs 19:298-307

4. Stapleton SJ, Holden J, Epstein J, Wilkie DJ (2016) Symptom clusters in patients with cancer in the hospice/palliative care setting. Support Care Cancer 24:3863-3871

5. Kane RL, Wales J, Bernstein L, Leibowitz A, Kaplan S (1994) A randomized controlled trial of hospice care. Lancet 1:890-894

6. Higginson IJ, Finlay IG, Goodwin DM, et al. (2003) Is there evidence that palliative care teams alter end-of-life experiences of patients and their caregivers? J Pain Symptom Manag e25:150-68

7. Mercadante S, Villari P, Ferrera P (2003) A model of acute symptom control unit: Pain Relief and Palliative Care Unit of La Maddalena Cancer Center. Support Care Cancer 11:114-119

8. Bercow AS, Nitecki R, Haber H et al (2021) Palliative care referral patterns and measures of aggressive care at the end of life in patients with cervical cancer. Int J Gynecol Cancer 31:66-72

9. Michael N, Beale G, O'Callaghan C et al (2019) Timing of palliative care referral and aggressive cancer care toward the end-of-life in pancreatic cancer: a retrospective, single-center observational study. BMC Palliat Care 18:13

10. Mercadante S, Intravaia G, Villaru P et al (2008) Clinical and financial analysis of an acute palliative care unit in an oncological department. Palliat Med 22:760-767

11. Ewing JA (1984) Detecting alcoholism The CAGE questionnaire 252:1905-1907

12. Breitbart W, Rosenfeld B, Roth A, et al. (1997) The Memorial Delirium Assessment Scale. J Pain Symptom Manage 13:128e137

13. Mercadante S, Coluzzi F (2021) Factors influencing pain expression in patients with cancer: an expert opinion. Pain Ther. https:// doi.org/10.1007/s40122-021-00272-x

14. Bruera E, Kuehn N, Miller MJ, Selmser P, Macmillan K. (1991) The Edmonton Symptom Assessment System (ESAS): a simple method for the assessment of palliative care patients. J Palliat Care 7:6e9

15. Mercadante S, Valle A, Sabba S, Casuccio A et al (2013) Pattern and characteristics of advanced cancer patients admitted to hospices in ItalyA. Support Care Cancer 21:935-939

16. Coym A, Ullrich A, Hackspiel LK et al (2020) Systematic symptom and problem assessment at admission to the palliative care ward - perspectives and prognostic impacts. BMC Palliat Care 19:75

17. West E, Pasman HR, Galesloot C, Lokker ME, OnwuteakaPhilipsen B (2016) Hospice care in the Netherlands: who applies and who is admitted to inpatient care? BMC Health Serv Res 28(16):33

18. Koorn R, van Klinken M, de Graaf E et al (2020) Are hospice patients and what care is provided in hospices? A pilot study. Palliat Med 37:448-454

19. Chung JH, Kim SH, Suh SY et al (2021) Differences between dedicated and not dedicated hospice physicians in symptoms and signs improvement among advanced cancer patients. Medicine 100:32

20. Cheng WW, Willey J, Palmer JL, Zhang T, Bruera E (2005) Interval between palliative care referral and death among patients treated at a comprehensive cancer center. J Palliat Med 8:1025-1032 
21. See D, Le B, Gorelik A, et al. (2019) Symptom burden in malignant and nonmalignant disease on admission to a palliative care unit. BMJ Supp Palliat Care Feb 4;bmjspcare-2018-001560.

22. Mercadante S, Adile C, Caruselli A et al (2016) The palliativesupportive care unit in a comprehensive cancer center as crossroad for patients' oncological pathway. PLoS One. 11:e0157300

23. Mercadante S, Fulfaro F, Casuccio A (2000) The impact of home palliative care on symptoms in advanced cancer patients. Support Care Cancer 8:307-310

24. Petracci E, Nanni L, Maltoni M et al (2016) Impact of admission to hospice on pain intensity and type of pain therapies administered. Support Care Cancer 24:225-232

25. Herr K, Titler M, Fine $P$ et al (2010) Assessing and treating pain in hospices: current state of Evidence-based practice. J Pain Symptom Manage 39:803-819

26. Koivu L, Polonen T, Stormi T, Salminen E (2014) End-of-life pain medication among cancer patients in hospice settings. Anticancer Res 34:6581-6584
27. Lammi UK, Kosunen E, Kellokumpu-Lehtinen P (2001) Palliative cancer care in two health centres and on hospice in Finland. Support Care Cancer 9:25-31

28. Heung Y, Azhar A, Naqvi SMAA et al (2021) Frequency and characteristics of first-time palliative care referrals during the last day of life. J Pain Symptom Manage 61:358-363

29. Bruera E, Hui D (2010) Integrating supportive and palliative care in the trajectory of cancer. Establishing goals and models of care. $\mathrm{J}$ Clin Oncol 28:5618-5623

30. Mercadante S, Gregoretti C, Cortegiani A (2018) Palliative care in intensive care units: why, where, what, who, when, how. BMC Anesthesiol 18:106

Publisher's note Springer Nature remains neutral with regard to jurisdictional claims in published maps and institutional affiliations. 